\title{
Stability of Blaschke's Characterization of Ellipsoids and Radon Norms
}

\author{
P. M. Gruber
}

Abteilung für Analysis, Technische Universität Wien, Wiedner Hauptstraße 8-10/1142, A-1040 Vienna, Austria pmgruber@pop.tuwien.ac.at

\begin{abstract}
Roughly speaking, in this article we prove quantitative versions of the following statements: (i) If each shadow boundary of a convex body in $\mathbb{E}^{d}(d \geq 3)$ under parallel illumination contains a curve which is almost planar and has the same shadow as the shadow boundary, then the body is approximately ellipsoidal. (ii) If for each diameter of a convex disk in $\mathbb{E}^{2}$ there is a diameter which is not far from being conjugate to the former, then the disk is close to a Radon disk. A consequence of these theorems is a stability result for the symmetry of orthogonality in finite-dimensional normed spaces. In addition, a stability result for the relation between different definitions of length in a normed plane is given.
\end{abstract}

\section{Introduction and Statement of Results}

1.1. How well can an object which almost satisfies a given condition, be approximated by objects satisfying it precisely? In convex geometry there are two kinds of pertinent results. First, the large body of stability results for geometric inequalities which can be traced back at least to Minkowski and Bonnesen. More recent systematic contributions are due to Volkov, Diskant, Fuglede, Groemer, Goodey, Schneider, and others, see Groemer's [11] survey. Second, a group of results of Groemer [9], [10], [12], [13], Goodey and Groemer [7], and Burger and Schneider [4] on the stability of geometric characterizations or properties of special convex bodies such as (solid) balls, ellipsoids, or prototiles. For an example see 2.7 below. In this article results of the second kind are considered.

1.2. Let $\mathcal{C}=\mathcal{C}\left(\mathbb{E}^{d}\right)$ be the space of all convex bodies in Euclidean $d$-space $\mathbb{E}^{d}$, that is, of all compact convex subsets of $\mathbb{E}^{d}$ with nonempty interior. For $d=2$ we speak of convex disks instead. On $\mathcal{C}$ the Pompeiu-Hausdorff metric $\delta^{\mathrm{H}}$ is defined as follows: let $C, D \in \mathcal{C}$, then $\delta^{\mathrm{H}}(C, D)$ is the maximum Euclidean distance which a point of one of the bodies $C, D$ can have from the other body. For each $C \in \mathcal{C}$ there is a unique ellipsoid 
$E(C)$ of minimum volume containing $C$, the minimum or Löwner ellipsoid of $C$. We let $B^{d}$ denote the (solid) Euclidean unit ball of $\mathbb{E}^{d}$.

A classical characterization of ellipsoids of Blaschke [2], [3], also proved by Herglotz presumably after Blaschke pointed it out to him, is the following: a convex body $C \in \mathbb{E}^{d}$ $(d \geq 3)$ is an ellipsoid if and only if for any parallel illumination the shadow boundary of $C$ is a planar convex "curve." (When speaking of "curves," we mean $(d-2)$-spheres.) Actually, Blaschke proved this result under additional smoothness assumptions, but a more precise version of his proof yields the general result. Marchaud [21], and others before him under the assumption of central symmetry, showed that it is sufficient to assume that each shadow boundary contains a planar convex curve with the same shadow. More precisely, this may be expressed in the following way: for any straight line $L$ through the origin $o$ there is a hyperplane $H$ such that

$$
C+L=(C \cap H)+L,
$$

where $A+B=\{x+y: x \in A, y \in B\}$ for $A, B \subset \mathbb{E}^{d}$. Blaschke's characterization of ellipsoids has been the subject of refinements and extensions both in convexity and differential geometry. It has many applications, the first being - in more modern terms - a characterization of Riemannian spaces among Finsler spaces, see [3]. This result answers for $d \geq 3$ a question of Carathéodory in the calculus of variations. The case $d=2$ was settled by Radon [24] using special norms which now are called Radon norms. For references and pertinent results see [15]-[17] and [23]. The following result is a stability version of the above characterization of ellipsoids.

Theorem 1. Let $C \in \mathcal{C}\left(\mathbb{E}^{d}\right)(d \geq 3)$ with $E(C)=B^{d}$ and let $\varepsilon>0$. Assume that for any line $L$ through o there is a slab $P$ of width $2 \varepsilon$ (measured in the direction of $L$ ) such that

$$
C+L=(C \cap P)+L
$$

Then

$$
\delta^{\mathrm{H}}(C, E(C)) \leq \alpha \varepsilon^{1 / 4},
$$

where the constant $\alpha>0$ depends on d only.

The assumption of this result means that each shadow boundary of $C$ contains a curve with the same shadow as the shadow boundary which is contained in a slab of width $2 \varepsilon$.

Tools for the proof are John's theorem on minimal ellipsoids, well-known properties of support functions and absolutely continuous functions, Gronwall's lemma, a result of Ewald et al. on line-segments in the boundary of convex bodies, and, finally, Groemer's stability result refining one of Brunn's characterizations of ellipsoids.

1.3. Let $\mathcal{C}_{o}=\mathcal{C}_{o}\left(\mathbb{E}^{d}\right)$ be the space of $o$-symmetric convex bodies. For $C, D \in \mathcal{C}_{o}$ their Banach-Mazur distance $\delta^{\mathrm{BM}}(C, D)$ is $\inf \{\lambda \geq 1: C \subset l(D) \subset \lambda C$ for suitable linear $l: \mathbb{E}^{d} \rightarrow \mathbb{E}^{d}$ \}. Note that $\delta^{\mathrm{BM}}$ is symmetric in $C, D$ and it is invariant with respect to linear transformations of $C, D$. Applying it to unit balls of norms on $\mathbb{E}^{d}$, the common Banach-Mazur distance for norms on $\mathbb{E}^{d}$ results. A plane supports a convex body $C$ (at a boundary point) if it meets the boundary (at this point) but not the interior of $C$. A 
diameter of a convex disk $D \in \mathcal{C}\left(\mathbb{E}^{2}\right)$ is a chord of $C$ with the property that there are parallel support lines of $C$ passing through its endpoints. For $D \in \mathcal{C}_{o}\left(\mathbb{E}^{2}\right)$ we stipulate that a diameter contains $o$.

A convex disk $D \in \mathcal{C}_{o}\left(\mathbb{E}^{2}\right)$ is called a Radon disk, its boundary a Radon curve, and the norm with unit disk $D$ a Radon norm if it has the property of conjugate diameters: for each pair $S, T$ of diameters of $D$ such that $T$ is parallel to support lines of $D$ at the endpoints of $S$, also $S$ is parallel to support lines at the endpoints of $T$. The standard construction of Radon disks is presented in 2.8 below. Radon disks and norms were introduced by Radon [24]. Their importance lies in the fact that many properties which for $d \geq 3$ hold only for ellipsoids or Euclidean norms, in case $d=2$ are valid precisely for Radon disks or norms, respectively. For a list of such properties see [16].

By the property of conjugate diameters up to $\varepsilon>0$ of a convex disk $D \in \mathcal{C}_{o}\left(\mathbb{E}^{2}\right)$ we mean the following: if $S, T$ are diameters of $D$ such that $T$ is parallel to support lines of $D$ at the endpoints of $S$, then there is a diameter $U$ of $D$ parallel to support lines of $D$ at the endpoints of $T$ and the distance of an endpoint of $U$ to the nearest endpoint of $S$ is $<\varepsilon$. Here distance is with respect to the norm with unit disk $D$. This notion is used in the following result on the stability of the definition of Radon disks.

Theorem 2. Let $D \in \mathcal{C}_{o}\left(\mathbb{E}^{2}\right)$ and $\varepsilon>0$ be given. Assume that $D$ has the property of conjugate diameters up to $\varepsilon$. Then there is a Radon disk $R \in \mathcal{C}_{o}\left(\mathbb{E}^{2}\right)$ such that

$$
\delta^{\mathrm{BM}}(D, R) \leq 1+\beta \varepsilon
$$

where $\beta>0$ is an absolute constant.

1.4. Theorems 1 and 2 may be used to derive stability versions of certain consequences of Blaschke's characterization of ellipsoids and of properties of Radon norms; we present two.

A vector $x \in \mathbb{E}^{d}$ is orthogonal to a vector $y \in \mathbb{E}^{d}$ with respect to a given norm $\|\cdot\|$ on $\mathbb{E}^{d}$, in symbols $x \perp y$, if $\|x\| \leq\|x+\lambda y\|$ for all $\lambda \in \mathbb{R}$. Carathéodory's problem referred to in 1.2 amounts to specifying all norms on $\mathbb{E}^{d}$ with symmetry of orthogonality; that is, for any $x, y$ with $x \perp y$, also $y \perp x$ holds. As shown by Radon [16] and Blaschke [3] this is precisely the case if $d=2$ and the norm is Radon, or if $d \geq 3$ and the norm is Euclidean.

Given a norm $\|\cdot\|$ on $\mathbb{E}^{d}$, the corresponding notion of orthogonality is symmetric up to $\varepsilon>0$ if it has the following property: for all $x, y \in \mathbb{E}^{d}$ such that $\|x\|=\|y\|=1$ and $x \perp y$, there is a point $z \in \mathbb{E}^{d}$ with $\|z\|=1,\|z-x\| \leq \varepsilon$ for which $y \perp z$. Using this concept, the following result is a direct consequence of Theorems 1 and 2 .

Corollary 1. Let $\|\cdot\|$ be a norm on $\mathbb{E}^{d}$ and $\varepsilon>0$. Assume that the corresponding notion of orthogonality is symmetric up to $\varepsilon$. Then there is a norm $|\cdot|$ on $\mathbb{E}^{d}$ such that

$$
\delta^{\mathrm{BM}}(\|\cdot\|,|\cdot|) \leq 1+\gamma \varepsilon^{p},
$$

where in case $d=2$ the norm $|\cdot|$ is Radon and $p=1$, while for $d \geq 3$ the norm $|\cdot|$ is Euclidean and $p=\frac{1}{4}$. The constant $\gamma>0$ depends only ond. 
The following result is a special case both of a result of Gruber [14] (see also [26]) and a result of Goodey and Woodcock [8] and Goodey [6]: let $C$ be a convex body in $\mathbb{E}^{d}(d \geq 3)$ such that bd $C \cap \operatorname{bd}(C+x)$ is contained in a hyperplane for each $x \in \mathbb{E}^{d}$ different from $o$. Then $C$ is an ellipsoid. Here bd stands for boundary. (In Gruber's result $C$ is assumed to be a compact connected set while Goodey and Woodcock and Goodey consider two convex bodies $C$ and $D$ and assume that bd $C \cap \operatorname{bd}(D+x)$ is contained in a hyperplane for all $x$ for which $C \neq D+x$. Gruber, Goodey and Woodcock, and Goodey give references to various related results.) A stability version of the above is the following easy consequence of Theorem 1 .

Corollary 2. Let $C \in \mathcal{C}\left(\mathbb{E}^{d}\right)(d \geq 3)$ with $E(C)=B^{d}$ and let $\varepsilon>0$. Assume that for each $x \in \mathbb{E}^{d}, x \neq o$, there is a slab $P$ of width $\varepsilon$ (measured in the direction of $x$ ) such that

$$
\text { bd } C \cap \operatorname{bd}(C+x) \subset P \text {. }
$$

Then

$$
\delta^{H}(C, E(C)) \leq \alpha \varepsilon^{1 / 4},
$$

where the constant $\alpha>0$ depends only on $d$.

1.5. We add a simple stability result for Radon norms.

Consider a norm $\|\cdot\|$ on $\mathbb{E}^{d}$ with unit ball $B$, say, and let $V(\cdot)$ denote the ordinary volume in $\mathbb{E}^{d}$. Then the length of a vector $x$ may be defined in the following two ways: first, of course, as $\|x\|$ and second, following Minkowski [22], by

$$
\|x\|_{M}=\lim _{\varepsilon \rightarrow+0} \frac{V([o, x]+\varepsilon B)}{\varepsilon^{d-1}},
$$

where $[o, x]$ denotes the line segment with endpoints $o$ and $x$. Actually, $\|\cdot\|_{M}$ is a norm on $\mathbb{E}^{d}$. The question arises of characterizing the norms $\|\cdot\|$ for which these two concepts of length coincide (after renormalizing the volume suitably, if necessary). Radon [24] proved that for $d=2$ it is precisely the Radon norms that have this property. In case $d \geq 3$ we conjecture that this property characterizes Euclidean norms; see [16]. The following theorem is a stability version of Radon's result.

Theorem 3. Let $\|\cdot\|$ be a norm on $\mathbb{E}^{2}$ and $\varepsilon>0$. Assume that, after a suitable renormalization of the area in $\mathbb{E}^{2}$, if necessary,

$$
\left|\|x\|-\|x\|_{M}\right| \leq \varepsilon \quad \text { for all } \quad x \in \mathbb{E}^{2} \text { with }\|x\|=1 .
$$

Then there is a Radon norm $|\cdot|$ on $\mathbb{E}^{2}$ such that

$$
\delta^{\mathrm{BM}}(\|\cdot\|,|\cdot|) \leq 1+\delta \varepsilon
$$

where $\delta>0$ is an absolute constant.

Explicit values of the constants $\alpha, \ldots, \delta$ may be obtained from an inspection of the tools and the proofs of our results. 


\section{Tools and Preliminaries}

2.1. A well-known result of John [19] is the following:

(1) Let $C \in \mathcal{C}$ be such that $E(C)=B^{d}$. Then $(1 / d) B^{d} \subset C \subset B^{d}$.

2.2. An easy continuity argument yields the next proposition.

(2) For any interior point of a convex disk there is a diameter containing the point.

2.3. The support function $h:[0,2 \pi] \rightarrow \mathbb{R}$ of a convex disk $D \in \mathcal{C}\left(\mathbb{E}^{2}\right)$ is defined by $h(\varphi)=\max \{x \cdot(\cos \varphi, \sin \varphi): x \in D\}$ where $\varphi \in[0,2 \pi]$ and the dot denotes the ordinary inner product in $\mathbb{E}^{2}$. Clearly, for each $\varphi \in[0,2 \pi], L(\varphi)=\{x \in$ $\left.\mathbb{E}^{2}: x \cdot(\cos \varphi, \sin \varphi)=h(\varphi)\right\}$ is the support line of $D$ with exterior normal unit vector $(\cos \varphi, \sin \varphi)$. The following proposition contains several well-known properties of support functions:

(3) Let $D \in \mathcal{C}\left(\mathbb{E}^{2}\right)$ and let $h$ be its support function. Then $h$ is absolutely continuous and differentiable for all $\varphi \in[0,2 \pi]$ with at most a countable set of exceptions. If $h^{\prime}(\varphi)$ exists, then the support line $L(\varphi)$ meets $D$ at a single point and $h^{\prime}(\varphi)$ is the (signed) distance (positive in the counterclockwise direction, negative otherwise) of the foot of $o$ on $L(\varphi)$ and the intersection point $D \cap L(\varphi)$.

(This follows from the fact that, in essence, $h$ is the restriction to the unit circle $S^{1}$ of a homogeneous convex and thus Lipschitz function on $\mathbb{E}^{2}$, from Corollary 1.7.3 in [27], the fact that the boundary of a convex disk contains at most countably many line segments, and from Theorem 1.7.4 in [27].)

2.4. The following tools deal with absolutely continuous functions; see, e.g., [28].

(4) If $f, g:[0,2 \pi] \rightarrow \mathbb{R}$ are absolutely continuous and $g>0$, then $f / g$ is also absolutely continuous.

(5) If $f:[0,2 \pi] \rightarrow \mathbb{R}$ is absolutely continuous, then

$$
f(\varphi)=f(0)+\int_{0}^{\varphi} f^{\prime}(\psi) d \psi \quad \text { for each } \varphi \in[0,2 \pi] .
$$

2.5. The following result is Gronwall's lemma, see [18]:

(6) Let $f$ be a real-valued continuous function on the interval $[\kappa, \lambda]$ such that

$$
0 \leq f(\varphi) \leq \mu+v \int_{\kappa}^{\varphi} f(\psi) d \psi \quad \text { for each } \varphi \in[\kappa, \lambda]
$$

where $\mu, v>0$ are suitable constants. Then

$$
f(\varphi) \leq \mu e^{\nu(\varphi-\kappa)} \quad \text { for each } \quad \varphi \in[\kappa, \lambda] .
$$

2.6. Let $S^{d-1}$ denote the Euclidean unit sphere in $\mathbb{E}^{d}$. Given $C \in \mathcal{C}\left(\mathbb{E}^{d}\right)$, the shadow boundary $S(u)$ of $C$ under illumination parallel to $u \in S^{d-1}$ consists of all boundary 
points of $C$ at which there are support lines of $C$ parallel to $u$. $S(u)$ is sharp if it does not contain a line segment parallel to $u$. By a result of Ewald et al. [5] we have the following:

(7) Let $C \in \mathcal{C}\left(\mathbb{E}^{d}\right)$. Then $S(w)$ is sharp for almost all $w \in S^{d-1}$.

2.7. We now state in a form suited for our purpose a stability version, due to Groemer [12], of one of Brunn's characterizations of ellipsoids.

(8) Let $C \in \mathcal{C}\left(\mathbb{E}^{d}\right)$ with $E(C)=B^{d}$ be given and let $\tau>0$. Assume that for each $u \in S^{d-1}$ the set of midpoints of all chords of $C$ parallel to $u$ is contained between two hyperplanes of the form $G(u) \pm \tau u$. Then

$$
\delta^{\mathrm{H}}(C, E(C)) \leq \sigma \tau^{1 / 4},
$$

where the constant $\sigma>0$ depends on $d$ only.

2.8. In this subsection we first describe the standard construction of Radon disks and then list some simple properties of these.

Consider a convex arc $A$ (on the boundary of a convex disk with center $o$ ) which is contained in an $o$-symmetric parallelogram $P$ and connects the midpoints of two consecutive edges of $P$. Choose a coordinate system and a Euclidean norm $\|\cdot\|_{2}$ such that $P$ is the unit square and $A$ connects the points $(1,0)$ and $(0,1)$. For each $x \in A$ consider its polar line with respect to the unit circle $S^{1}$ (of the norm $\|\cdot\|_{2}$ ). Rotate this line about $o$ by the angle $\pi / 2$ in the positive direction. The lines thus obtained envelop a convex $\operatorname{arc} A^{\#}$ in $P$ which connects the points $(0,1)$ and $(-1,0)$. The $\operatorname{arcs} A, A^{\#}$ and their reflections in $o$ together determine a Radon curve and thus a Radon disk, say $R$. Each Radon disk can be obtained in this way; see [20] and [24].

The image of a Radon disk under a nonsingular linear transformation is again a Radon disk. Well-known properties of polarity and the above construction yield the following propositions, where the width of a convex disk in a given direction is the ordinary width of the smallest slab orthogonal to this direction which contains the disk:

(9) Let $A$ and $R$ be as above and let $x$ be a smooth point of $A$. Then the corresponding rotated polar line of $x$ meets $A^{\#}$ and thus $R$ at a single point and conversely.

(10) Let $A, R$ and $\|\cdot\|_{2}$ be as above and let $x \in A$. Then the width of $R$ in the direction orthogonal to $x$ is $2\|x\|_{2}^{-1}$.

2.9. A result of Reidemeister [25], proved in a more precise form by Anderson and Klee [1], yields our last tool.

(11) Let $C \in \mathcal{C}_{o}\left(\mathbb{E}^{d}\right)$. Then for almost all $u \in S^{d-1}$ a suitable positive multiple of $u$ is a smooth point of the boundary of $C$.

2.10. A general reference for convex geometry is [27]. 


\section{Proof of Theorem 1}

Let $C$ be the convex body of Theorem 1 . Choosing $\alpha$ large enough we see that it is clearly sufficient to prove the theorem under the assumption that

(12) $0<\varepsilon \leq 1 / d(d+1)$.

3.1. The main step of the proof is to show that $C$ satisfies the assumptions of Groemer's stability result (8):

(13) Let $u \in S^{d-1}$. Then the set of all midpoints of chords of $C$ parallel to $u$ is contained between two hyperplanes, say $G(u) \pm \varrho \varepsilon u$, where $\varrho>0$ is a constant depending only on $d$.

For $u \in S^{d-1}$ let $H(u) \pm \varepsilon u$ be the boundary hyperplanes of the slab $P=P(u)$, which corresponds to the line through $o$ and $u$.

The proof of (13) is divided into several steps.

3.2. First, we show the following simple proposition, where $\Varangle$ stands for angle:

(14) $\tan (\pi / 2-\Varangle(w, H(w))) \leq d+1$ for each $w \in S^{d-1}$.

Let the superscript $w$ denote the orthogonal projection of $\mathbb{E}^{d}$ onto the subspace orthogonal to $w$ of codimension 1. By the assumption of the theorem, $C^{w}=(C \cap P(w))^{w}$. Together with (1) this shows that

$$
(1 / d) B^{d w} \subset C^{w}=(C \cap P(w))^{w} \subset\left(B^{d} \cap P(w)\right)^{w} \subset B^{d w} .
$$

Now, noting that the slice $B^{d} \cap P(w)$ is contained between the hyperplanes $H(w) \pm \varepsilon w$ and thus has width at most $2 \varepsilon \leq 2 / d(d+1)$, see (12), the inclusions (15) together with a simple geometric argument yield (14).

3.3. Secondly, we prove a weak form of (13):

(16) Let $u \in S^{d-1}$ be such that the shadow boundary $S(u)$ is sharp. Let $[p, q]$ be a chord of $C$ parallel to $u$. Then the midpoint of $[p, q]$ is contained between the two hyperplanes $H(u) \pm \varrho \varepsilon u$, where $\varrho>0$ is a constant which depends only on $d$.

In this subsection we suppose that

$$
d=3
$$

otherwise consider a section of $C$ with a three-dimensional subspace containing $u$ and $[p, q]$.

Clearly, the assumption of Theorem 1 implies the following:

(17) Let $w \in S^{2}$. Then, if a support line of $C$ parallel to $w$ meets $C$ at one point only, this point is contained between the hyperplanes $H(w) \pm \varepsilon w$.

3.3.1. We now need some preparations: $p^{u}=q^{u}$ is an interior point of the convex disk $C^{u}$. Thus, by (2), there is a diameter $\left[s^{u}, t^{u}\right]$ of $C^{u}$ containing this point where $s$ and $t$ 
are suitable points of $S(u)$. Clearly, there are support planes of $C$ at $s$ and $t$ which are parallel to each other and to $u$ and thus also to $[p, q]$. Let $H$ be the plane parallel to these support planes and containing $[p, q]$. Choose a Cartesian coordinate system in $H$ with origin $o=[s, t] \cap[p, q]=[s, t] \cap H$ and let $h:[0,2 \pi] \rightarrow[0,+\infty)$ be the support function of the convex disk $D=C \cap H$. It follows from $o \in D$ that $h \geq 0$.

Since $S(u)$ is sharp (see (16)),

(18) the support plane of $C$ at $s \in S(u)$ parallel to $H$ has only $s$ or a line segment containing $s$ in common with $C$. An analogous remark holds for $t$.

3.3.2. Next, the following will be shown:

(19) $0 \leq h(\varphi) \leq(4 \pi \varepsilon+m) e^{2 \pi(d+1)}$ for each $\varphi \in[0,2 \pi]$, where $m=\min \{h(\psi): \psi \in$ $[0,2 \pi]\}$.

By suitably rotating the Cartesian coordinate system in $H$ about $o$, if necessary, we may suppose that

(20) $h(0)=m$.

For the proof of (19) the main step is to verify the following statement:

(21) Let $\psi \in[0,2 \pi]$ be such that $h^{\prime}(\psi)$ exists and $w=(-\sin \psi, \cos \psi)$ is not parallel to a line segment as described in (18). Then

$$
h^{\prime}(\psi) \leq 2 \varepsilon+(d+1) h(\psi)
$$

To show this, we first prove that

(22) $\xi \varepsilon w \in H \cap H(w)$ for a suitable $\xi \in[-1,1]$.

Since $H$ is parallel to support planes of $C$ at $s$ and $t$ and since $w \in H$, there are support lines of $C$ at $s$ and $t$, respectively, which are parallel to $w$. By our choice of $\psi$, and thus of $w$, in (21), they intersect $C$ only at $s$ and $t$, respectively. Hence (17) implies that

$$
s+\sigma \varepsilon w, t+\tau \varepsilon w \in H(w) \quad \text { for suitable } \sigma, \tau \in[-1,1] .
$$

Now, noting that $o \in[s, t]$ (see 3.3.1), (22) follows. Next we show that

(23) $h(\psi) v+h^{\prime}(\psi) w+\eta \varepsilon w \in H \cap H(w)$ for a suitable $\eta \in[-1,1]$, where $v=(\cos \psi, \sin \psi)$.

By (3), $h^{\prime}(\psi)$ is the signed distance of the line through $o$ and $v$ to the unique point of $D$ where the support line of $D$ with exterior normal vector $v$ (and thus parallel to $w$ ) intersects $D$. Hence this point is $h(\psi) v+h^{\prime}(\psi) w$. (17) then yields (23). By (14) the tangent of the angle $\vartheta$ between the line $H \cap H(w)$ and the line through $o$ and $v$ is at most $d+1$. Hence (22) and (23) together imply

$$
h^{\prime}(\psi)+\eta \varepsilon-\xi \varepsilon \leq \tan \vartheta \cdot h(\psi) \leq(d+1) h(\psi)
$$

Since $\xi, \eta \in[-1,1]$ this in turn yields the inequality in (21). 
To finish the proof of (19), we proceed as follows: by (3), $h$ is absolutely continuous. Thus (5) together with (21) shows that

$h(\varphi)-h(0)=\int_{0}^{\varphi} h^{\prime}(\psi) d \psi \leq 4 \pi \varepsilon+(d+1) \int_{0}^{\varphi} h(\psi) d \psi \quad$ for each $\varphi \in[0,2 \pi]$.

Applying Gronwall's lemma (6) and taking into account (20), the proof of (19) is concluded.

3.3.3. Similar arguments lead to the following proposition:

(24) $|h(\varphi)-h(\varphi+\pi)| \leq 8 \pi e^{2 \pi(d+1)} \varepsilon$ for each $\varphi \in[0,2 \pi]$.

After a suitable rotation of the Cartesian coordinate system in $H$ about $o$, if necessary, we can assume that

(25) $h(0)=h(\pi)$.

As before, the main step is to show that the following holds:

(26) Let $\psi \in[0,2 \pi]$ be such that $h^{\prime}(\psi)$ and $h^{\prime}(\psi+\pi)$ exist and $w=(-\sin \psi, \cos \psi)$ is not parallel to a line segment as described in (18). Then

$$
\left|h^{\prime}(\psi)-h^{\prime}(\psi+\pi)\right| \leq 4 \varepsilon+(d+1)|h(\psi)-h(\psi+\pi)| .
$$

Let $v=(\cos \psi, \sin \psi)$. As in the proof of (19) it is shown that

(27) $\xi \varepsilon w, h(\psi) v+h^{\prime}(\psi) w+\eta \varepsilon w,-h(\psi+\pi) v-h^{\prime}(\psi+\pi) w-\zeta \varepsilon w \in H \cap H(w)$ for suitable $\xi, \eta, \zeta \in[-1,1]$.

Let $\vartheta$ be the angle between the line $H \cap H(w)$ and the line through $o$ and $v$. Then (27) implies

$$
\begin{aligned}
h^{\prime}(\psi)+\eta \varepsilon-\xi \varepsilon & =\tan \vartheta \cdot h(\psi), \\
\xi \varepsilon+h^{\prime}(\psi+\pi)+\zeta \varepsilon & =\tan \vartheta \cdot h(\psi+\pi) .
\end{aligned}
$$

Hence

$$
\begin{aligned}
\left|h^{\prime}(\psi)-h^{\prime}(\psi+\pi)+(\eta-2 \xi-\zeta) \varepsilon\right| & =|\tan \vartheta| \cdot|h(\psi)-h(\psi+\pi)| \\
& \leq(d+1)|h(\psi)-h(\psi+\pi)|
\end{aligned}
$$

by (14). Since $\xi, \eta, \zeta \in[-1,1]$ this yields (26).

The remaining part of the proof of (24) makes use of (3), (5), (26), (6), and (25). It is analogous to the proof of the last part of (19).

3.3.4. In the final step of the proof of (16) we distinguish two cases.

Case 1. Assume that

$$
m \leq 4 \pi \varepsilon
$$

Then (19) shows that

$$
0 \leq h(\varphi) \leq 8 \pi e^{2 \pi(d+1)} \varepsilon \quad \text { for each } \quad \varphi \in[0,2 \pi]
$$


and thus

$$
D \subset 8 \pi e^{2 \pi(d+1)} \varepsilon B^{2} .
$$

Hence the midpoint of the line segment $[p, q] \subset D$ parallel to $u$ has distance at most $8 \pi e^{2 \pi(d+1)} \varepsilon$ from $o \in[p, q]$. By the assumption of (16) the shadow boundary $S(u)$ is sharp. Thus an application of (17) with $w=u$ shows that the points $s, t(\in S(u))$ and therefore also the point $o \in[s, t]$ (compare 3.3.1) are between the planes $H(u) \pm \varepsilon u$. Taken together this yields (16) in the first case.

Case 2. Assume that

$$
m \geq 4 \pi \varepsilon .
$$

Then the definition of $m$ and (19) show that

$$
m \leq h(\varphi) \leq 2 e^{2 \pi(d+1)} m \quad \text { for each } \varphi \in[0,2 \pi] .
$$

Thus

(28) $m B^{2} \subset D \subset 2 e^{2 \pi(d+1)} m B^{2}$.

We may suppose that $\|p\|_{2} \leq\|q\|_{2}$ where $\|\cdot\|_{2}$ is the given Euclidean norm on $\mathbb{E}^{2}$. $-p$ is a boundary point of $-D$, and $q$ and $-p$ are on the same ray starting at $o$, but $q$ is farther away from $o$. Consider a support line of $D$ parallel to and on the far side of a support line of $-D$ at $-p$. Then $q$ is between these support lines. By (24) the distance between these support lines is at most $8 \pi e^{2 \pi(d+1)} \varepsilon$. Now, taking into account (28), an elementary argument shows that

$$
\|q-(-p)\|_{2}=\|p+q\|_{2} \leq 16 \pi e^{4 \pi(d+1)} \varepsilon .
$$

The same argument as in the proof of the first case then concludes the proof of (16) in the second case.

3.4. In 3.3 we have proved a weak version of (13), see (16). Using it we can now easily finish the proof of (13):

Let $u \in S^{d-1}$. If $S(u)$ is sharp, then putting $G(u)=H(u)$, it is seen that proposition (13) reduces to (16). If $S(u)$ is not sharp, we may choose a sequence $u_{1}, u_{2}, \ldots \in S^{d-1}$ by (2) which converges to $u$ and such that each shadow boundary $S\left(u_{i}\right)$ is sharp. Since the corresponding hyperplanes $H\left(u_{i}\right)$ all satisfy (14) and meet $C$ or, at least, have distance at most $\varepsilon$ from $C$, the following holds: by taking a suitable subsequence and renumbering it, if necessary, we may suppose that the sequence $H\left(u_{1}\right), H\left(u_{2}\right), \ldots$ converges (in the natural way) to a hyperplane $G(u)$, say. Now, since (16) holds for $u_{i}$ and $H\left(u_{i}\right)$, a simple limiting argument shows that the set of all midpoints of chords of $C$ parallel to $u$ is contained between the two hyperplanes $G(u) \pm \varrho \varepsilon u$. This concludes the proof of (13) in case $S(u)$ is not sharp. Thus the proof of (13) is complete.

3.5. By (13), $C$ satisfies the assumptions of Groemer's stability result (8). Hence

$$
\delta^{\mathrm{H}}(C, E(C)) \leq \sigma \varrho^{1 / 4} \varepsilon^{1 / 4},
$$

concluding the proof of Theorem 1. 


\section{Proof of Theorem 2}

Let $D$ be the convex disk of Theorem 2. As in the proof of Theorem 1, it can be assumed for the proof of Theorem 2 that

(29) $0<\varepsilon<\frac{1}{11}$.

4.1. To the convex disk $D$ we assign a Radon disk $R$ in the following way: Consider an $o$-symmetric parallelogram of minimum area which contains $D$. The midpoints of its edges are contained in $D$; otherwise there is an $o$-symmetric parallelogram of smaller area which still contains $D$. Now choose a coordinate system and a (new) Euclidean norm, say $\|\cdot\|_{2}$, such that the parallelogram becomes the unit square. Then, using the boundary arc of $D$ in the positive quadrant which connects the points $(1,0)$ and $(0,1)$ construct $R$ as described in 2.8 .

This construction shows that

(30) $(1 / \sqrt{2}) B^{2} \subset D, R \subset \sqrt{2} B^{2}$.

Let $h$ and $k$ denote the support functions of $D$ and $R$, respectively, and let $\|\cdot\|$ be the norm with unit disk $D$.

4.2. The following proposition is the core of the proof:

(31) $|h(\varphi) / k(\varphi)-1| \leq \pi \varepsilon$ for each $\varphi \in[0,2 \pi]$.

To verify it we first show the following:

(32) Let $\psi \in(\pi / 2, \pi)$ be such that $h^{\prime}(\psi)$ and $k^{\prime}(\psi)$ exist. Then $\left|(h(\psi) / k(\psi))^{\prime}\right| \leq$ $2 \varepsilon$.

Let $N$ be the line through $o$ which subtends the angle $\psi$ with the positive first coordinate axis.

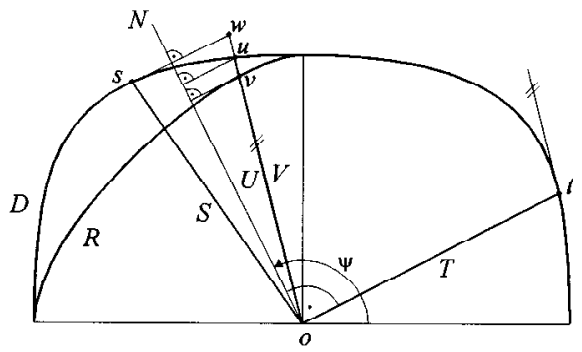

Let $s$ and $v$ be the points where the support lines of $D$ and $R$ with exterior normal unit vector $(\cos \psi, \sin \psi)(\in N)$ meet $D$ and $R$, respectively. Since $h^{\prime}(\psi)$ and $k^{\prime}(\psi)$ exist, these points are unique and

(33) $h^{\prime}(\psi)$ is the signed distance of $s$ and its foot on $N$, $k^{\prime}(\psi)$ is the signed distance of $v$ and its foot on $N$ 
(see (3)). Let $S$ be the diameter of $D$ having one endpoint $s$ and let $T$ be the diameter of $D$ orthogonal to $N$. Denote by $t$ its endpoint in the positive quadrant. By construction, $T$ is parallel to a support line of $D$ at the endpoint $s$ of $S$ and, similarly, parallel to a support line of $R$ at $v$. Since $k^{\prime}(\psi)$ exists, (3) implies that the latter support line intersects $R$ only at $v$. Hence, by (9), $t$ is a smooth point of $R$ and thus of $D$. Using the fact that by construction $R$ has the property of conjugate diameters, the diameter $U$ of $D$ parallel to the supporting line of $D$, respectively $R$ at $t$, then is contained in the same line as $V$. Let $u$ be the endpoint of $U$ in the upper half-plane and let

$$
w=\frac{h(\psi)}{k(\psi)} v .
$$

Since the line segment connecting $s$ and $w$ is parallel to $T$ and since the line through $o$ and $w$ (which contains both $V$ and $U$ ) is parallel to the support line of $D$ at $t$, we see that $\|s-w\| \leq\|s-u\|$. The property that $D$ has conjugate diameters up to $\varepsilon$ yields $\|s-u\| \leq \varepsilon$. These remarks together with (30) show that $\|s-w\|_{2} \leq \sqrt{2} \varepsilon$. Using (33), the latter inequality can be expressed in the form

$$
\left|h^{\prime}(\psi)-\frac{h(\psi)}{k(\psi)} k^{\prime}(\psi)\right| \leq \sqrt{2} \varepsilon .
$$

Since, according to (30), $k(\psi) \geq 1 / \sqrt{2}$, it thus follows that

$$
\left|\frac{h^{\prime}(\psi) k(\psi)-h(\psi) k^{\prime}(\psi)}{k(\psi)^{2}}\right| \leq 2 \varepsilon,
$$

concluding the proof of (32).

Having proved (32), we can easily prove (31): $h$ and $k$ are absolutely continuous by (3). Since $k \geq 1 / \sqrt{2}$, proposition (4) implies that $h / k$ is absolutely continuous. By construction, $h(\pi / 2)=k(\pi / 2)=1$. Together with (32) and (5) this shows that

$$
\begin{aligned}
\left|\frac{h(\varphi)}{k(\varphi)}-1\right| & =\left|\int_{\pi / 2}^{\varphi}\left(\frac{h(\psi)}{k(\psi)}\right)^{\prime} d \psi\right| \leq \int_{\pi / 2}^{\varphi}\left|\left(\frac{h(\psi)}{k(\psi)}\right)^{\prime}\right| d \psi \\
& \leq 2 \varepsilon \frac{\pi}{2}=\pi \varepsilon \quad \text { for } \varphi \in\left[\frac{\pi}{2}, \pi\right] .
\end{aligned}
$$

This finishes the proof of (31) for $\varphi \in[\pi / 2, \pi]$ and thus, by the symmetry of $D$ and $R$ in $o$, also for $\varphi \in[3 \pi / 2,2 \pi]$. Since $h(\varphi)=k(\varphi)$ for $\varphi \in[0, \pi / 2] \cup[\pi, 3 \pi / 2]$ by construction of $R$, the proof of (31) is complete.

4.3. Since, by (31),

$$
(1-\pi \varepsilon) k \leq h \leq \frac{1+\pi \varepsilon}{1-\pi \varepsilon}(1-\pi \varepsilon) k
$$

we obtain that

or

$$
(1-\pi \varepsilon) R \subset D \subset \frac{1+\pi \varepsilon}{1-\pi \varepsilon}(1-\pi \varepsilon) R
$$

$$
\delta^{\mathrm{BM}}(D, R)=\delta^{\mathrm{BM}}(R, D)=\delta^{\mathrm{BM}}((1-\pi \varepsilon) R, D) \leq \frac{1+\pi \varepsilon}{1-\pi \varepsilon} \leq 1+10 \varepsilon,
$$

where we have made use of (29). This concludes the proof of Theorem 2. 


\section{Proof of Corollary 1}

5.1. Assume first that

$$
d=2 \text {. }
$$

Let $D$ be the unit disk of the given norm $\|\cdot\|$. By definition, two diameters $S$ and $T$ of $D$ are conjugate up to $\varepsilon$ if and only if their endpoints $s$ and $t$, respectively, exhibit symmetry of orthogonality up to $\varepsilon$. Hence the case $d=2$ of Corollary 1 is an immediate consequence of Theorem 2 .

5.2. We now assume that

$$
d \geq 3 \text {. }
$$

Let $C$ be the solid unit ball of the norm $\|\cdot\|$. Obviously it is sufficient to prove the corollary for

(34) $0<\varepsilon \leq \min \left\{1 / d(d+1), 1 / 32 \alpha^{4} d^{2}(d+1)\right\}$.

Choose a Euclidean norm $\|\cdot\|_{2}$ such that $E(C)=B^{d}$, where $B^{d}$ is the solid unit ball of the norm $\|\cdot\|_{2}$. Then (1) implies

(35) $(1 / d) B^{d} \subset C \subset B^{d}=E(C)$.

We show that the symmetry of orthogonality up to $\varepsilon$ with respect to $\|\cdot\|$ yields the following proposition:

(36) Let $u \in S^{d-1}$ be such that a positive multiple of $u$, say $y$, is a smooth boundary point of $C$. Then there is a subspace $H(u)$ of codimension 1 with the property that $S(u)$ is contained between the hyperplanes $H(u) \pm d^{2}(d+1) \varepsilon u$, and so that

$$
\tan \left(\frac{\pi}{2}-\Varangle(u, H(u))\right) \leq d(d+1) .
$$

Let $y+H(u)$ be the unique support hyperplane of $C$ at $y$. Let $x \in S(u)$. Since $y$ is a multiple of $u$, we have $x \perp y$. Hence by the symmetry of orthogonality up to $\varepsilon$ there is a boundary point $z$ of $C$ with $y \perp z$ and $\|x-z\| \leq \varepsilon$. Since $y$ is a smooth boundary point of $C$ and $y+H(u)$ is the unique support hyperplane of $C$ at $y$, each $z$ with $y \perp z$ is contained in the subspace $H(u)$. These remarks show that the distance between $x$ and $H(u)$ with respect to the norm $\|\cdot\|$ is $\leq \varepsilon$. Observing that $x$ was an arbitrary point of $S(u)$ and taking into account (35) we obtain that $S(u)$ is contained between the two hyperplanes parallel to and at $\left(\|\cdot\|_{2}-\right)$ distance $d \varepsilon$ from $H(u)$. This, together with (34), (35), and a simple geometric argument, implies that

$$
\tan \left(\frac{\pi}{2}-\Varangle(u, H(u))\right) \leq d(d+1)
$$

(compare a similar argument in 3.2). This in turn yields that $S(u)$ is contained between hyperplanes of the form $H(u) \pm d^{2}(d+1) \varepsilon u$. Thus, the proof of (36) is complete. 
An immediate consequence of (11), (35), and (36) is the following assertion:

(37) $E(C)=B^{d}$ and there is a dense set $S$ in $S^{d-1}$ such that the following hold: for $u \in S$ there is a slab $P(u)$ of width $2 d^{2}(d+1) \varepsilon$ (measured in the direction of u) such that

$$
\tan \left(\frac{\pi}{2}-\Varangle(u, P(u))\right) \leq d(d+1)
$$

and

$$
C+L=(C \cap P(u))+L,
$$

where $L$ is the line through $o$ and $u$.

By an argument similar to that used in 3.4 we may omit the restriction on $u$ in (37). Thus $C$ satisfies the assumption of Theorem 1. Hence, as a consequence of (34), we have

$$
\delta^{\mathrm{H}}\left(C, B^{d}\right) \leq \alpha\left(2 d^{2}(d+1)\right)^{1 / 4} \varepsilon^{1 / 4}=\sigma \varepsilon^{1 / 4} \leq \frac{1}{2},
$$

where the constant $\sigma>0$ depends only on $d$. Thus

$$
\left(1-\sigma \varepsilon^{1 / 4}\right) B^{d} \subset C \subset E(C)=B^{d},
$$

or, equivalently,

$$
\delta^{\mathrm{BM}}\left(C, B^{d}\right) \leq \frac{1}{1-\sigma \varepsilon^{1 / 4}} \leq 1+\gamma \varepsilon^{1 / 4}
$$

for a suitable constant $\gamma>0$ depending only on $d$. This concludes the proof of Corollary 1 in case $d \geq 3$.

\section{Proof of Corollary 2}

We clearly may assume that

(38) $0<\varepsilon<1 / d(d+1)$.

6.1. From (1) it follows that

(39) $(1 / d) B^{d} \subset C \subset B^{d}=E(C)$.

For each $u \in S^{d-1}$, let $H(u)$ and $H(u)+\varepsilon u$ be the boundary hyperplanes of the slab corresponding to $x=\varepsilon u$.

We now show the following:

(40) Let $u \in S^{d-1}$ be such that the shadow boundary $S(u)$ is sharp. Then $S(u)$ is contained between the two hyperplanes $H(u) \pm \varepsilon u$.

For the remainder of this proof, when speaking of "above" or "below" this is meant with respect to the direction of $u$. Since by (38) and (39), $C \cap(C+\varepsilon u)$ has nonempty interior, $H(u)$ is not parallel to $u$. By the assumption of Corollary 2, the set bd $C \cap$ $\operatorname{bd}(C+\varepsilon u)$ is above $H(u)$ and below $H(u)+\varepsilon u$. Since $S(u)$ is sharp this yields that 
the shadow $S(u)+\varepsilon u$ of $C+\varepsilon u$ is above $H(u)$ and the shadow boundary $S(u)$ of $C$ is below $H(u)+\varepsilon u$. Hence $S(u)$ is between $H(u) \pm \varepsilon u$, concluding the proof of (40).

(38), (39), and (40) together with an elementary argument show:

(41) Let $u$ be as in (40). Then $\tan (\pi / 2-\Varangle(u, H(u))) \leq d+1$.

6.2. Combining (39), (40), and (41), we see that

(42) $E(C)=B^{d}$ and there is a dense set $S$ in $S^{d-1}$ such that for any $u \in S$ there is a slab $P(u)$ of width $2 \varepsilon$ (measured in the direction of $u$ ), such that

$$
\tan \left(\frac{\pi}{2}-\Varangle(u, P(u))\right) \leq d+1
$$

and

$$
C+L=(C \cap P(u))+L,
$$

where $L$ is the line through $o$ and $u$.

By an argument similar to that used in 3.4 the restriction on $u$ can be omitted in (42). Thus $C$ satisfies the assumption of Theorem 1 and an application of the latter yields

$$
\delta^{\mathrm{H}}\left(C, B^{d}\right) \leq \alpha \varepsilon^{1 / 4}
$$

concluding the proof of Corollary 2.

\section{Proof of Theorem 3}

We may assume that

(43) $0<\varepsilon \leq \frac{1}{6}$.

7.1. Let $D$ be the unit disk of the given norm $\|\cdot\|$. Choose a Euclidean norm $\|\cdot\|_{2}$ and construct a Radon disk $R$ as in 4.1. Let $|\cdot|$ be the Radon norm with unit disk $R$. By construction,

(44) $(1 / \sqrt{2}) B^{2} \subset D, R \subset \sqrt{2} B^{2}$.

Let $h$ and $k$ be the support functions of $D$ and $R$, respectively, using the inner product corresponding to $\|\cdot\|_{2}$.

7.2. By construction,

(45) $h(\varphi)=k(\varphi)$ for each $\varphi \in[0, \pi / 2]$.

The next step is to show that

(46) $|h(\varphi)-k(\varphi)|<4 \varepsilon$ for each $\varphi \in[\pi / 2, \pi]$. 
Let $x$ with $\|x\|=1$ be a positive multiple of the unit vector $(\cos (\varphi-\pi / 2)$, $\sin (\varphi-\pi / 2))$. By the construction in 4.1 and 2.8, proposition (10), and the respective definitions of $\|\cdot\|_{M}$ and $|\cdot|_{M}$ in 1.5 , we have

(47) $\|x\|_{M}=h(\varphi)\|x\|_{2} \cdot \tau$,

$$
(1 / \tau)|x|_{M}=k(\varphi)\|x\|_{2}=\left(1 /\|x\|_{2}\right)\|x\|_{2}=1=|x|=\|x\|,
$$

where $\tau>0$ is a constant such that the area is suitably normalized. (47), (44), and the assumption of Theorem 3 show that

(48) $|\tau h(\varphi)-k(\varphi)|=\left.\left|\|x\|_{M}-(1 / \tau)\right| x\right|_{M}\left|\left(1 /\|x\|_{2}\right) \leq\right|\|x\|_{M}-\|x\| \mid \sqrt{2} \leq \sqrt{2} \varepsilon$.

In particular, if $\varphi=\pi / 2$ we have $h(\pi / 2)=k(\pi / 2)=1$ and therefore $|\tau-1| \leq \sqrt{2} \varepsilon$. This, (48), and (44) imply that

$$
\begin{aligned}
|h(\varphi)-k(\varphi)| & \leq|\tau h(\varphi)-k(\varphi)|+|\tau-1| h(\varphi) \\
& \leq \sqrt{2} \varepsilon+\sqrt{2} \varepsilon \sqrt{2}<4 \varepsilon,
\end{aligned}
$$

which concludes the proof of (46).

Since $D$ and $R$ are symmetric in $o$, it follows from (4) and (46) that

$$
\delta^{\mathrm{H}}(D, R)=\max \{|h(\varphi)-k(\varphi)|: \varphi \in[0,2 \pi]\}<4 \varepsilon,
$$

where $\delta^{\mathrm{H}}$ is defined with respect to the Euclidean norm $\|\cdot\|_{2}$ and we have used a common representation of $\delta^{\mathrm{H}}$ by means of support functions. Hence, using (44) we obtain

$$
(1-4 \sqrt{2} \varepsilon) D \subset R \subset(1+4 \sqrt{2} \varepsilon) D
$$

or, noting (43),

$$
\delta^{\mathrm{BM}}(\|\cdot\|,|\cdot|)=\delta^{\mathrm{BM}}(D, R) \leq \frac{1+4 \sqrt{2} \varepsilon}{1-4 \sqrt{2} \varepsilon} \leq 1+\delta \varepsilon
$$

for a suitable constant $\delta>0$. Thus, the proof of Theorem 3 is finished.

\section{Acknowledgments}

I am obliged to Professors Groemer and Schnitzer for valuable hints. The figure was drawn by Dr. Reitzner.

\section{References}

1. Anderson, R. D., Klee, V., Convex functions and upper semi-continuous collections, Duke Math. J. 19 (1952), 349-357.

2. Blaschke, W., Kreis und Kugel, Göschen, Leipzig, 1916, de Gruyter, Berlin, 1956.

3. Blaschke, W., Räumliche Variationsprobleme mit symmetrischer Transversalitätsbedingung, Ber. Verh. Sächs. Akad. Wiss. Math.-naturw. Kl. 68 (1916), 50-55; Ges. Werke, vol. 3, pp. 167-172, Thales-Verlag, Essen, 1985. 
4. Burger, T., Schneider, R., On convex bodies close to ellipsoids, J. Geom. 47 (1993), 16-22.

5. Ewald, G., Larman, D., Rogers, C. A., The direction of line segments and of the $r$-dimensional balls on the boundary of a convex body in Euclidean space, Mathematika 17 (1970), 1-20.

6. Goodey, P. R., Homothetic ellipsoids, Math. Proc. Cambridge Philos. Soc. 93 (1983), 25-34.

7. Goodey, P. R., Groemer, H., Stability results for first order projection bodies, Proc. Amer. Math. Soc. 109 (1990), 1103-1114.

8. Goodey, P. R., Woodcock, M. M., Intersection of convex bodies with their translates, in: The Geometric Vein (The Coxeter Festschrift), pp. 289-296, Springer-Verlag, New York, 1981.

9. Groemer, H., On convex bodies that permit packings of high density, Discrete Comput. Geom. 5 (1990), 357-364.

10. Groemer, H., Stability theorems for projections and central symmetrizations, Arch. Math. 56 (1991), 394399.

11. Groemer, H., Stability of geometric inequalities, in: P. M. Gruber, J. M. Wills, eds., Handbook of Convex Geometry, vol. A, pp. 125-150, North-Holland, Amsterdam, 1993.

12. Groemer, H., Stability theorems for ellipsoids and spheres, J. London Math. Soc. (2) 49 (1994), 357-370.

13. Groemer, H., Stability results for convex bodies and related spherical integral transformations, $A d v$. in Math. 109 (1994), 45-74.

14. Gruber, P. M., Über kennzeichnende Eigenschaften von Ellipsoiden und euklidischen Räumen II, J. Reine Angew. Math. 270 (1974), 123-142.

15. Gruber, P. M., Über kennzeichnende Eigenschaften von Ellipsoiden und euklidischen Räumen III, Monatsh. Math. 78 (1974), 311-340.

16. Gruber, P. M., Radons Beiträge zur Konvexität/Radon's contributions to convexity, in: J. Radon, Ges. Abh., vol. I, pp. 331-342, Birkhäuser, Basel, 1987.

17. Gruber, P. M., Höbinger, J., Kennzeichnungen von Ellipsoiden mit Anwendungen, in: Jahrb. Überbl. Math. 1976, pp. 9-29, Bibliographisches Institut, Mannheim, 1976.

18. Hartmann, P., Ordinary Differential Equations, Wiley, New York, 1964.

19. John, F., Extremum problems with inequalities as subsidiary conditions, in: Studies and Essays Presented to R. Courant, pp. 187-204, Interscience, New York, 1948.

20. Leichtweiss, K., Konvexe Mengen, Springer-Verlag, Berlin, 1980, VEB Deutsch. Verl. Wiss., Berlin, 1980.

21. Marchaud, A., Un théoreme sur les corps convexes, Ann. Sci. École Norm. Sup. (3) 76 (1959), 283-304.

22. Minkowski, H., Über die Begriffe Länge, Oberfläche und Volumen, Jahresber. Deutsch. Math.-Verein. 9 (1901), 115-121; Ges. Abh., vol. II, pp. 122-127, Teubner, Leipzig, 1911.

23. Petty, C., Ellipsoids, in: P. M. Gruber, J. M. Wills, eds., Convexity and Its Applications, pp. 264-276, Birkhäuser, Basel, 1983.

24. Radon, J., Über eine besondere Art ebener konvexer Kurven, Ber. Verh. Sächs. Akad. Wiss. Math.-naturw. Kl. 68 (1916), 123-128; Ges. Abh., vol. I, 361-366, Birkhäuser, Basel, 1987.

25. Reidemeister, K., Über die singulären Randpunkte eines konvexen Körpers, Math. Ann. 83 (1921), 116118.

26. Šaidenko, A. V., Some characteristic properties of the ellipsoid, Sibirsk. Mat. Zh. 21 (1980), 232-234, 240.

27. Schneider, R., Convex Bodies: The Brunn-Minkowski Theory, Cambridge University Press, Cambridge, 1993.

28. Vinogradov, I. M. et al., eds., Encyclopedia of Mathematics, English translation, Kluwer, Dordrecht, 19881994.

Received May 28, 1996, and in revised form September 5, 1996. 